\title{
Innovación pedagógica reflexiva en comunidades profesionales de aprendizaje y su impacto en la formación docente institucional
}

\section{Reflective pedagogical innovation in professional learning communities and its impact on institutional teacher training}

\author{
Federico Malpica Basurto ${ }^{1}$, Pedro Navareño Pinadero ${ }^{2}$ \\ ${ }^{1}$ Instituto Escalae, España (fmalpica@escalae.org) \\ ${ }^{2}$ Instituto Escalae, España (pnavareno@escalae.org)
}

\section{RESUMEN:}

El objetivo de este artículo es reflexionar sobre el impacto que la innovación pedagógica reflexiva en comunidades profesionales de aprendizaje tiene en la formación docente institucional, en nuestro caso, a través de un análisis y evaluación de carácter cuantitativo y cualitativo, que realizamos de las opiniones expresadas por los usuarios de la plataforma online Teachers Pro $^{1}$, y que exponemos como resultados del desarrollo y aplicación de esta plataforma, considerada como herramienta para el desarrollo profesional que se adapta y responde a las necesidades formativas docentes, tanto individuales como institucionales, y que busca la creación y desarrollo de comunidades profesionales de aprendizaje docente, conformada por todos sus usuarios.

Para ello partimos de la idea de innovación pedagógica reflexiva, pues consideramos que debe ser la esencia y base en la que se asiente la organización y el funcionamiento de las instituciones educativas, como medio de garantizar, que sus finalidades educativas y las acciones colectivas que se realizan para alcanzar sus objetivos sean la brújula que guíe la nave desde una acción pedagógica innovadora, colectiva, creativa, responsable, comprometida y sistemática de todos sus miembros,

\footnotetext{
${ }^{1}$ TeachersPro es una plataforma de aprendizaje docente online
} basada en los últimos descubrimientos científicos sobre la ofreciendo así la respuesta más adecuada que, en cada momento y contexto, demanda una sociedad global, interconectada y dinámica.

PALABRAS CLAVE: INNOVACIÓN PEDAGÓGICA REFLEXIVA, PLANIFICACIÓN ESTRATÉGICA, COMUNIDADES PROFESIONALES DE APRENDIZAJE, FORMACIÓN DOCENTE INSTITUCIONAL, DESARROLLO PROFESIONAL DOCENTE.

\begin{abstract}
:
The objective of this article is to reflect on the impact that reflective pedagogical innovation in professional learning communities has on institutional teacher training, in our case, through a quantitative and qualitative analysis and evaluation, which we carry out of the opinions expressed by users of the Teachers Pro online platform, and which we present as results of the development and application of this platform, considered as a tool for professional development that adapts to and responds to the educational training needs, both individual and institutional, and which seeks creation and development of professional teacher learning communities, made up of all its users.

For this we start from the idea of reflective pedagogical innovation, because we believe that it
\end{abstract}

transferencia de la teoría a la práctica profesional: http://www.teacherspro.com/ 
should be the essence and basis on which the organization and operation of educational institutions is based, as a means of guaranteeing that their educational purposes and the collective actions that they are made to achieve their objectives, be the compass that guides the ship from a collective innovative, creative, responsible, committed and systematic pedagogical action of all its members, offering the most appropriate response that, in every moment and context, a society demands due to its global, interconnected and dynamic nature.

KEYWORDS: REFLECTIVE PEDAGOGICAL INNOVATION, STRATEGIC PLANNING, PROFESSIONAL LEARNING COMMUNITIES, IN-SERVICE TEACHER TRAINING, PROFESSIONAL DEVELOPMENT.

\section{INTRODUCCIÓN}

Las organizaciones humanas están abocadas a enfrentarse a procesos de permanente actualización e innovación en sus métodos de trabajo, su organización y su funcionamiento, así como en los objetivos y contenidos a desarrollar para atender las nuevas demandas sociales que un mundo tan dinámico, globalizado e interconectado exige, cada vez más, y de un modo más acelerado. Este contexto, de constantes cambios e incertidumbre, demanda nuevas formas de planificación estratégica y de innovación pedagógica, que deben partir de dos competencias docentes básicas: una, la formación de la competencia reflexiva del profesorado para mejorar su capacidad de análisis sobre la práctica, que le permita construir en cada momento la respuesta que se ajuste mejor a sus necesidades y, la segunda, imprescindible y complementaria de la anterior, la capacidad de investigar de manera rigurosa $y$ sistemática en y sobre la realidad institucional (interna) y social (externa) en la que interviene. Todo ello como garantía de conocer para, creativamente, encontrar la respuesta más adecuada. Asimismo, entendemos como imprescindible desarrollar la competencia colaborativa de cada docente, que le permita reflexionar con sus iguales, compartir la práctica, aprender unos de otros y generar un mayor criterio profesional al analizar otras respuestas a problemas y situaciones comunes. Además, entendemos que dicho proceso debe estar sistematizado en las instituciones educativas, mediante un plan estratégico de innovación y mejora pedagógica sostenible, que permita a los docentes avanzar de forma gradual a partir del desarrollo de acuerdos metodológicos comunes, que les ayuden a caminar por la senda de la eficiencia, logrando que el impacto de los planes estratégicos en la innovación pedagógica reflexiva contribuya eficazmente a la formación docente institucional como motor de desarrollo del bien común, tal y como se plantea en el Informe de la Unesco (2015).

Este sistema de formación docente, centrado en atender las finalidades educativas y en un eficiente funcionamiento institucional, deberá partir de las necesidades reales de los propios docentes, en una dinámica de abajo hacia arriba, tomando en cuenta los objetivos institucionales, como un generador de conocimiento compartido, basado en la capacidad de reflexividad, tanto individual como colectiva, así como de un sistema abierto y flexible que permita desarrollar sinergias, a partir del compromiso y la colaboración de sus miembros, que contribuyan de manera positiva $y$ real a un funcionamiento institucional, que, por tanto, se base en la creación y consolidación de hábitos docentes colectivos en comunidades profesionales de aprendizaje o comunidades para la institucionalización de la práctica educativa (Malpica, 2013).

Tal importancia tiene este aspecto que podríamos afirmar lo siguiente: el futuro será de aquellas organizaciones que encuentren la forma de articular una cultura organizacional basada en la capacidad institucional de diseñar e implementar sus planes estratégicos desde la reflexividad y la colaboración de sus miembros, que les permita un desarrollo profesional y organizativo eficaz y adecuado a cada contexto.

En consecuencia, con este trabajo pretendemos indagar sobre cómo afectan las comunidades profesionales de aprendizaje en el desarrollo de la innovación pedagógica reflexiva, desarrollando espacios de formación colaborativa online para los docentes, que respondan a sus intereses y a las necesidades institucionales. Para ello hemos analizado las respuestas que los docentes han emitido, una vez han realizado una primera experiencia de desarrollo profesional en la plataforma TeachersPro, sobre cómo valoran su eficacia para responder a sus necesidades formativas, que contribuirá a su desarrollo profesional docente, a la vez que a lograr las finalidades educativas institucionales. Se analiza la conveniencia del desarrollo de las comunidades profesionales de aprendizaje, ya que la formación docente tradicional basada en el individualismo ha demostrado su ineficacia para formar al alumnado como personas con habilidades y valores bien afianzados, que solo se pueden alcanzar con la acción colaborativa y coordinada de todos los que trabajan 
en su formación, para mejorar la calidad de sus aprendizajes con la consistencia necesaria.

\section{ANTECEDENTES Y FUNDAMENTACIÓN TEÓRICA DE LA INNOVACIÓN PEDAGÓGICA REFLEXIVA Y COLABORATIVA}

\subsection{Antecedentes}

Todo profesional ha desarrollado, consciente o inconscientemente, un porcentaje de su práctica a través de la interacción con otros colegas. A veces, de manera informal participando en algún proyecto, en alguna discusión intentando dar solución a problemas concretos o como parte de un equipo que intenta realizar una mejora o innovación. La mayoría de las profesiones ya no se pueden entender sin estos espacios de trabajo colegiado, altamente supervisados, que les permita sentirse seguros en la aplicación de su práctica, pero también comportarse como estrategas, intentar prácticas nuevas y ser reconocidos por ellas.

Lo interesante para desarrollar una innovación pedagógica reflexiva y fundamentada sería que dejara de ser algo gobernado por el azar, la coincidencia y el voluntarismo de los propios profesionales, para transformarse en un modelo de formación $\mathrm{y}$ desarrollo docente bien estructurado, planificado, trabajado y evaluado.

Actualmente, entre los profesionales de la educación, el trabajo colaborativo no se da por añadidura ni por defecto, sobre todo porque no es la cultura heredada ni existe costumbre de compartir y crear conocimiento entre colegas. En realidad, lo común es que cada uno tenga su espacio de desarrollo y cada uno ejerza la docencia según sus propios referentes y herramientas didácticas.

Por tanto, lograr el desarrollo de un aprendizaje entre iguales es una conquista a la cual debería aspirar toda institución que quiera innovar en la práctica educativa.

No puede haber innovación en instituciones fraccionadas, que no sean capaces de generar conocimiento pedagógico común, ya que una condición indispensable de la mejora y la innovación es que pueda garantizar cierta consistencia en el proceso de enseñanza-aprendizaje que siguen los estudiantes al pasar por las diferentes aulas y profesorado a lo largo de toda su formación, y esto pasa por un aprendizaje colectivo de los docentes que forman parte de una misma institución educativa.

Este concepto de consistencia obliga a pensar en un proceso formativo que sea institucional y capaz de generar una metodología común entre el profesorado.
Por ello, necesitamos forzosamente un modelo que implique a todo el cuerpo docente. Sin embargo, hoy día la mayoría de la formación docente tradicional carece de dicha consistencia, porque son actividades formativas que no están pensadas para realizar una transferencia a todas las aulas mediante la práctica entre iguales y la reflexión conjunta, sino para volcar unos contenidos en ciertos docentes que se supone que, posterior a la formación recibida, deberán implementar y trasponer en sus aulas además de convencer al resto, pero que, de acuerdo a la experiencia, sucede en contadas ocasiones.

Tampoco basta con desarrollar algún grupo de mejora o equipo de innovación, ya que dichas estructuras mantienen pocas interrelaciones entre ellos. Forma cada uno su espacio de trabajo, pero no están necesariamente alineados con un propósito general que los pueda abarcar a todos. Son grupos nacidos a partir de iniciativas personales que no necesariamente persiguen un objetivo institucional. En cuanto a los equipos de trabajo, tienen un objetivo en común, que incluso puede ser concebido por la institución, pero que es solo de ese equipo y, por tanto, la experiencia y práctica necesaria para el cambio solo la desarrollan unos cuantos que, se supone, luego explicarán y convencerán al resto de realizar la misma experiencia, pero sabemos que normalmente esto último no sucede. La explicación puede ser que los demás colegas no han tenido la experiencia y, por tanto, no se ha generado en ningún momento la empatía necesaria ni la urgencia por el cambio, necesarias para institucionalizar hábitos docentes colectivos.

Superar el individualismo que caracteriza nuestra profesión no es tarea fácil. Requiere el desarrollo de estructuras adecuadas donde cada docente se sienta implicado, así como una metodología de trabajo que permita formarse en el diálogo reflexivo y en la colaboración con otros. También requiere compartir ciertos valores y objetivos comunes, que van más allá de la propia práctica personal. "Hablamos de comunidad cuando, además de compartir preocupaciones comunes, compartimos valores profesionales y sociales; cuando nuestra aportación no sólo es material, sino que trasciende otros ámbitos y llega hasta el compromiso" (Gairín Sallán, 2015, p. 18). Por tanto, la comunidad profesional de aprendizaje es aquella que se compone de profesionales que comparten una misma visión de lo que quieren conseguir en cuanto al aprendizaje de sus estudiantes, de tal manera que todos sus objetivos, proyectos y formación continua, contribuyen a esta visión compartida.

Cuando se desarrolla una comunidad profesional de aprendizaje, los diferentes equipos de mejora están 
interrelacionados por un referente común, que es transversal a todos ellos, y que permite que cada uno de estos equipos de mejora contribuya a dicho referente, que no es otro que la consecución de las finalidades del aprendizaje, es decir, mantener y mejorar la coherencia entre aquello que esperamos de los estudiantes cuando acaben su formación y lo que hacemos en todas las aulas para que cada uno alcance este perfil competencial.

Una comunidad profesional de aprendizaje consagra cada uno de los equipos que la integran a estudiar nuevas formas de cumplir cada vez mejor con sus finalidades del aprendizaje. Impulsa a los docentes a pensar creativamente sobre su práctica, y a cómo hacer para compartirla con el resto de los colegas. Cuando se encuentra una nueva práctica, se prueba $y$, si la evidencia comprueba su utilidad, se extiende al resto de colegas a través de redes de aprendizaje entre iguales. Cada equipo de mejora y los grupos que lo conforman, están pensando constantemente en diferentes aspectos de la práctica educativa, de una manera sistemática, organizada y alineada con un propósito común.

En este sentido, el proceso de construcción y desarrollo de las comunidades profesionales de aprendizaje ha de ser cíclico. Cada período debería pasar por una socialización por parte del colectivo docente acerca de los fines de la educación y del aprendizaje que se pretende conseguir en sus estudiantes. Una vez resuelta esta cuestión y clarificados los objetivos educativos comunes, la siguiente pregunta es: ¿cómo está contribuyendo cada docente al perfil competencial de los estudiantes? La respuesta a esta pregunta merece un autodiagnóstico periódico de la práctica educativa por parte de cada profesional (quien primero que debe darse cuenta de sus necesidades y carencias) y, con base en los datos obtenidos sobre la realidad de las aulas, la elaboración de un plan estratégico para fortalecer las capacidades pedagógicas colectivas en función del perfil competencial que se quiere conseguir en el alumnado, el cual ayude a enfocar las ideas, discusiones, acciones de mejora, proyectos de innovación y decisiones sobre inversiones o adecuaciones del centro.

Después de estos ejercicios, los grupos de mejora tienden a evolucionar porque ya no se manejan de forma independiente, sino que comienzan a pensar en soluciones para problemas comunes, encontrados a partir de un análisis participado por todo el profesorado, donde el aprendizaje entre iguales es un elemento indispensable. Por tanto, el autodiagnóstico pedagógico y el plan estratégico para fortalecer capacidades pedagógicas son el elemento que detona la evolución de los grupos y equipos de mejora para construir una comunidad profesional de aprendizaje docente.

Siguiendo este proceso participativo y reflexivo, tanto en la definición de las finalidades de aprendizaje y el análisis de la práctica educativa, como en la planificación estratégica del desarrollo docente, quedaría ahora hacer realidad el plan definido para desarrollar la metodología común de la institución, contando con las aportaciones y compromiso de todo el profesorado (o al menos de una parte mayoritaria). Para ello, es importante que las acciones para desarrollar la práctica educativa y la formación docente no partan solo desde la dirección (o incluso de fuera del centro), sino que sean desarrolladas por los propios docentes, que deben hacer un trabajo de diseño de acuerdos metodológicos entre colegas que, posteriormente, se conviertan en el contenido sobre el cual se desarrolle la práctica entre iguales, el aprendizaje y la evaluación.

Si la formación y desarrollo docente giran en torno a los acuerdos generados por el propio profesorado, sobre aquello necesario para garantizar el perfil competencial que pretende lograr la institución en sus estudiantes, se genera un aprendizaje con mayor sentido, que resulta más significativo, profundo y funcional para los profesionales, capaz de producir con mayores garantías una mejora continua y fundamentada de la práctica educativa, que dé sentido a los proyectos de mejora y de innovación pedagógica en las aulas.

\subsection{Fundamentación teórica de la planificación estratégica para una innovación pedagógica reflexiva}

Cuando las metas institucionales no están definidas y acordadas, o solo lo están sobre el papel, sin estar siendo aplicadas en la práctica, cualquier acción educativa puede ser considerada adecuada. $\mathrm{Si}$, por el contrario, tenemos acordadas nuestras finalidades educativas y queremos ser coherentes con ellas, estamos obligados a desarrollar y a poner en práctica planes estratégicos de innovación que nos permitan dar la respuesta más adecuada en cada momento y contexto a nuestros estudiantes. Pero ese camino, que es fácil de resumir, sin embargo, está lleno de obstáculos y dificultades de todo tipo, como ya hemos señalado anteriormente y la experiencia nos enseña. Tal es así que, en estos momentos, podemos considerar como una necesidad de primer orden el que las instituciones educativas dispongan de planes de actuación que puedan proyectar en el tiempo las acciones priorizadas e integrar, unificando criterios, los diferentes proyectos o planes particulares de 
actuación, con el fin de vertebrar la tarea colectiva institucional.

Si analizamos la planeación desde su significado podríamos decir que

consiste en definir las metas de la organización, establecer una estrategia general para alcanzarlas y trazar planes exhaustivos para integrar y coordinar el trabajo de la organización. La planificación se ocupa tanto de los fines (qué hay que hacer) como de los medios (cómo hay que hacerlo). (Robbins y Coulter, 2005, p. 158)

Aunque la planificación estratégica tiene su origen en el ámbito militar, fue a mediados de siglo pasado cuando en diferentes contextos empresariales se desarrolló como herramienta y "como instrumento de apoyo a la gestión pública en el marco de las iniciativas del New Public Management, de mediados de los ochenta en los países de la OCDE" (Armijo, 2009, p. 6).

En muchos casos se ha asociado la planificación estratégica con el medio y largo plazo, quedando para el corto los planes de acción operativos, que concretan en un periodo de tiempo más corto, normalmente un año, el conjunto de acciones, sus responsables, los recursos, etc., así como los medios, instrumentos e indicadores de logro, que permitan conocer el grado y nivel alcanzado con relación a los objetivos o metas propuestos.

En el ámbito educativo español (y también en diversos países de Latinoamérica), desde los años ochenta y posteriores, se desarrollaron e hicieron obligatorios planes a medio y largo plazo como los proyectos educativos, donde debía participar toda la comunidad educativa, y los proyectos curriculares, responsabilidad del cuerpo docente, en los que se proyectaban las actuaciones a largo plazo para cada escuela. Por su parte, las acciones para un año escolar se recogían en las denominadas programaciones generales anuales (PGA) o planes anuales. En ellos se hacían explícitas las priorizaciones y acciones particulares que se llevarían a cabo durante ese curso escolar. Los resultados alcanzados y propuestos en dichas PGA deberían ser valorados en la memoria anual, que debía recoger un balance general de lo realizado durante el curso, incluido el análisis y la valoración de los resultados académicos de los estudiantes, además de establecer propuestas de mejora para ser recogidas en la PGA del curso siguiente, con el fin de dar continuidad y mejorar los procesos de gestión institucional de acuerdo con los planes a medio y largo plazo. Pero hay que decir que son escasos los centros en los que este proceso se hace con el rigor necesario para pensar que no se trata de una burocracia más.
Si entendemos por calidad educativa la respuesta sistemática, rigurosa y adecuada a cada momento que se ofrece a los estudiantes y a la sociedad, desde las instituciones educativas, para formar ciudadanos pacíficos, solidarios y responsables, con los principios éticos y valores necesarios, para construir una sociedad justa y sostenible, en la que la vida se rija por el respeto a los derechos de todas las personas, en ese caso debemos aceptar que el cambio vertiginoso del mundo actual nos irá exigiendo, al ritmo de los tiempos, desarrollar planes estratégicos de ajuste permanente y flexible a las nuevas demandas sociales, es decir, que también contengan elementos de innovación educativa para dar respuesta a estas demandas presentes y futuras.

También es cierto que hablar de innovación en educación no es aún un término generalizado, pues podríamos aceptar que la escuela es una de las instituciones más anquilosadas actualmente, a pesar la presencia de tecnologías, como afirman Senge Cambron-McCabe y Lucas (2002, p. 44) cuando dicen que "Sin embargo, habiendo pasado bastante tiempo con educadores y con personas de negocios, mi opinión es que los educadores se sienten más atrapados e imposibilitados para innovar". Lo que vendría a reforzar aquella vieja idea de que a menudo "la escuela enseña contenidos del siglo XIX con profesores del siglo XX a alumnos del siglo XXI" (Monereo y Pozo, 2001, p. 50), combinación de elementos que aún se hace más difícil articular, cuando nadie nos puede garantizar qué formación van a necesitar nuestros estudiantes dentro de una o dos décadas. Pues no queda lugar a dudas, como expone Imbernón (2004, p. 6) que "la institución educativa... y la profesión docente... deben cambiar radicalmente, deben convertirse en algo verdaderamente diferente, adecuado a los cambios vertiginosos que han sacudido el siglo XXI".

\section{EL IMPACTO DEL LAS COMUNIDADES PROFESIONALES DE APRENDIZAJE EN LA INNOVACIÓN PEDAGÓGICA REFLEXIVA Y EN LA FORMACIÓN DOCENTE INSTITUCIONAL}

Para centrar la mirada en nuestra reflexión, consideramos que la innovación pedagógica reflexiva tiene que ser el medio más adecuado para atender las necesidades educativas de los estudiantes de un mundo instalado en la incertidumbre y en el cambio vertiginoso, en el que la escuela se transforme en un espacio abierto y flexible de aprendizajes para todos, que sea la mejor garantía de que todo el profesorado 
contribuye de modo eficaz al logro de las finalidades educativas institucionales. Para ello se necesita, no solo la voluntad y la actitud positiva de los docentes, sino que también se hace imprescindible construir un sistema de desarrollo permanente del profesorado, centrado en la escuela, que ofrezca una formación flexible y adecuada a cada docente, en función de sus necesidades y de la innovación necesaria para contribuir al logro de las finalidades de aprendizaje que cada institución tenga establecido. $\mathrm{Y}$ es, en ese proceso de innovación pedagógica reflexiva, donde herramientas como TeachersPro se ofrecen para que el profesorado desarrolle su entorno personal de aprendizaje o PLE (Navareño y Rincón, 2017).

Consideramos que

el mejor camino de formación y desarrollo profesional docente es justamente la reflexión sistemática y crítica sobre la práctica, individual y colectiva, retroalimentada por el conocimiento que la comunidad científica aporta, en cada momento y contexto, en relación con la materia y la forma en la que se debe enseñar y aprender. (Navareño, 2015, p. 27)

$\mathrm{Y}$ ese ejercicio de práctica reflexiva podemos considerarlo como un proceso de investigación que sienta las bases para la autoformación, que debe iniciarse con una reflexión inicial sobre nuestra práctica, facilitada por la aplicación de los instrumentos y modelos que nos proporciona la práctica reflexiva (Domingo y Gómez, 2014), tanto individualmente como en compañía de otros, para identificar nuestros puntos fuertes y áreas de mejora, hecho que nos ofrecerá el conocimiento necesario para retroalimentar nuestra reflexión sobre la práctica y ayudarnos a encontrar la respuesta formativa que necesitamos a partir del conocimiento existente, para así poder avanzar y mejorar el desarrollo profesional docente.

Pero consideramos que toda esa reflexión y colaboración sobre la práctica debe sentarse sobre las bases de acuerdos metodológicos previos por parte de todo el profesorado del centro, para proponer una formación docente institucional de calidad, pertinente e innovadora que, a su vez, proponga un sistema de formación docente basado en la investigación como proceso de práctica reflexiva, o "construcción del conocimiento profesional docente como proceso de investigación-acción reflexiva" (Navareño, 2018, p. 52), que lleva al desarrollo de las comunidades profesionales de aprendizaje.

\section{DISEÑO Y METODOLOGÍA DEL ESTUDIO}

El estudio se realiza a partir de un sencillo diseño del análisis cualitativo y cuantitativo de las respuestas que los usuarios de la plataforma TeachersPro han ofrecido desde su puesta en funcionamiento el año 2016.

El método empleado para el análisis de datos cualitativos ha consistido en la recogida de las respuestas, agrupadas por similitud y regularidad, más frecuentes y representativas ofrecidas por los usuarios, que ponen de manifiesto la forma en la que valoran la realización de la formación correspondiente al desafío que cada uno ha efectuado. En cuanto al análisis cuantitativo ha consistido en recoger y analizar los porcentajes de frecuencias de las respuestas emitidas en relación con las cuestiones que consideramos planteadas.

\section{LA COMUNIDAD PROFESIONAL DE APRENDIZAJE ONLINE (TEACHERS PRO): ALGUNAS EVIDENCIAS}

El aprovechamiento y la aplicación de las TIC como herramienta didáctica, que conforma los llamados entornos digitales de aprendizaje, demanda que se opere un cambio radical en la concepción no solo de la enseñanza, sino también del aprendizaje, tanto en el alumnado como en el profesorado, ya que su aplicación en el aula, por parte de los docentes, plantea nuevos retos en la forma en la que se organiza la acción docente y la secuencia didáctica, pues además de la irrupción de esta potente herramienta, las aportaciones de las ciencias de la educación y, en concreto, las neurociencias, han venido a poner en evidencia algunas de las prácticas más arraigadas en la escuela, sobre todo las que hacen referencia al protagonismo que deben tener los estudiantes en su aprendizaje, frente a la pasividad que tenían en otros tiempos. Hoy sabemos que las metodologías activas deben ser el eje de la acción docente para mejorar la motivación intrínseca que produce la tarea bien hecha, lograda y graduada en pequeñas acciones de éxito. Por tanto, no es necesario recordar que la idea de aprendizaje basada en la acumulación de saberes cerrados y definidos, educación bancaria que denominaba Freire, hace tiempo que ha sido superada por una idea de aprendizaje más dinámico, abierto y centrada en competencias clave, que posibilitan la adquisición y el desarrollo las capacidades naturales, de aprender a aprender, que garanticen el aprendizaje a lo largo de toda la vida.

Con esa idea de escuela, abierta a nuevos saberes que surgen de manera permanente, consideramos que 
es más fácil de entender que la formación docente online haya adquirido una gran importancia y relevancia en el campo del desarrollo profesional docente, por sus muchas virtudes, entre ellas su gran accesibilidad, flexibilidad y su extraordinaria capacidad de adaptación a los intereses y a las necesidades formativas de cada uno, ya que permite su permanente actualización, como exige un mundo globalizado e interconectado.

En este contexto de aprendizaje docente, y de acuerdo con un sencillo método de análisis y evaluación de las respuestas de los usuarios de la plataforma TeachersPro, queremos recoger algunas evidencias, de las aportaciones que puede hacer una comunidad profesional de aprendizaje online como lo es esta plataforma que

está pensada y dirigida a mejorar la formación permanente de los docentes, mediante el aprendizaje y desarrollo de las capacidades, las habilidades y competencias de los profesores, a través del estudio de los Desafíos, que cada docente elige realizar y que se concretan en el estudio teórico práctico de tres habilidades en cada uno de ellos. Para lo cual la plataforma se adapta y propone a cada docente un itinerario formativo personalizado, según sus intereses y necesidades, en función de las respuestas que se dan a un cuestionario inicial. (Navareño y Rincón, 2017, p. 6)

En el estudio antes citado señalábamos que TeachersPro está diseñado para trabajar toda la competencia docente y, por tanto, la plataforma se caracteriza por ser: adaptativa, motivacional, gamificada, competencial, colaborativa y social.

Pero para comprobar y valorar su eficacia era necesario someter la plataforma online a que los docentes la utilizaran como medio de formación y, después, emitieran un juicio sobre ella. Los docentes completaron dos cuestionarios, uno sobre los desafíos con 8 preguntas ( 5 cerradas y 3 abiertas) y otro sobre habilidades con 9 preguntas (todas cerradas).

\section{DISCUSIÓN Y RESULTADOS}

La relevancia de los resultados obtenidos pone de manifiesto la gran importancia que tiene el ofrecer a los docentes la posibilidad de satisfacer sus necesidades formativas, pero con cierto equilibrio entre los intereses particulares y las necesidades de formación que la institución debe atender ante la demanda y las necesidades que presentan sus estudiantes. Pues la forma ideal de superar el eterno debate de cómo y para qué debemos enseñar será difícil de resolver si nos centramos en las visiones de cada docente. Por ello debe concretarse el cómo, qué y para qué enseñar centrando la mirada en las necesidades que demanda la sociedad y en las finalidades educativas que, en consecuencia, la comunidad educativa tiene acordadas en cada momento y en cada contexto.

De ahí la importancia de transformar la formación docente, para pasar de un modelo ya superado y obsoleto centrado en los intereses individuales del profesorado, que no está conectado con las necesidades institucionales y centrarnos en otro que atienda las necesidades formativas colegiadas que contribuyan a la creación de verdaderas comunidades profesionales de aprendizaje docente, dirigidas a dar la respuesta formativa que la comunidad educativa tiene acordada para sus estudiantes.

En ese sentido, los resultados obtenidos en este sencillo estudio vienen a mostrar que es posible formar a los docentes en ese doble sentido de atender lo individual y lo colectivo, pero siempre en función del interés general institucional.

Entre los resultados más importantes que hasta el momento hemos obtenido del análisis de los 308 desafíos y 2020 habilidades realizados desde que se abrió la plataforma, por docentes procedentes de más de 15 países (españoles y latinoamericanos en su gran mayoría, y, por tanto, de diferentes niveles y contextos sociales y educativos), podemos resumirlos en los siguientes resultados:

a) en relación con los desafíos:

1. La formación cumplió con las expectativas de aprender algo nuevo en un $78,25 \%$.

2. En un $70,78 \%$ la formación les ha revelado cosas sobre sí mismos y sobre cómo aprenden.

3. Esta formación les ha abierto nuevas perspectivas de mejora, en un $82,14 \%$ manifiesta que mucho o bastante.

4. La valoración global de la formación es positiva para un $78,57 \%$ de los docentes participantes.

5. A partir de la formación recibida el $69,49 \%$ de los docentes se sienten más seguros para enseñar a sus estudiantes.

6. Lo que más desean los docentes que perciban sus estudiantes de su modo de enseñar después de realizar el desafío es:

- Que perciban mi preocupación por enseñarles del mejor modo posible.

- Que he cambiado mi modo de enseñar, que ellos son los protagonistas, los actores del aprendizaje. 
- Que se puede aprender de otro modo diferente al tradicional.

- Que lo que aprenden les será de aplicación en la vida.

- Que la evaluación sea justa y adecuada a los aprendizajes.

- Que vean que tengo capacidad de empatía con ellos, que conecto y respondo a sus expectativas.

- Que perciban un ambiente de confianza, desarrollando una mejor autoestima y autoimagen, en el que todos aprendemos.

- Que se impliquen en su aprendizaje.

- Que potencien su capacidad creativa y el trabajo cooperativo.

- Que he terminado con el rodillo de enseñar lo que toca (libro de texto) siendo autocrítico con los contenidos y métodos, que los escucho, que los quiero y que son necesarios para el mundo.

- Que desarrollen herramientas para la comunicación y la interacción, que sus intereses y opiniones son tenidas en cuenta.

- Etc.

7. En cuanto a cómo el desafío puede ayudar a mis estudiantes a aprender mejor, el profesorado se expresa del modo siguiente:

- Porque aplico a la práctica lo aprendido con una correcta planificación.

- Porque incide en su motivación, su ilusión por aprender y participar en el aprendizaje, facilitando la investigación y la cooperación.

- Porque observo que aprenden habilidades con sentido y las aplican a lo concreto.

- Porque formamos grupos heterogéneos donde se pueden ayudar entre sí.

- Porque ellos son protagonistas de su aprendizaje.

- Porque partimos de sus intereses.

- Porque aplico mejor la metodología.

- Porque consigo que ellos gestionen mejor su aprendizaje.

- Porque les permite ver la aplicación práctica de lo que aprenden.

- Porque he podido aplicar actividades que he observado a otros compañeros de TeachersPro.

- Porque aplico habilidades aprendidas en el desafío, a través de las evidencias he aprendido mucho de otros compañeros y muchas de estas ideas las incorporaré en mi práctica educativa.

- Este desafío ayuda a que los estudiantes trabajen de forma colaborativa, encontrado soluciones a problemas reales y partiendo de lo que ellos saben y lo que no saben, buscando diferentes soluciones que podrán aplicar luego en diferentes contextos. Les va a ayudar a ser más críticos y a ser conscientes de su propio aprendizaje.

- Etc.

8. En relación con lo que consideran que se han beneficiado de la interacción con otros colegas a través de la comunidad de TeachersPro lo expresan del siguiente modo:

- Al compartir experiencias te das cuenta de que siempre hay cosas por descubrir y perfeccionar.

- Todos se enriquecen mucho más, he podido conocer la forma de trabajar de otros compañeros lo cual me ha servido tanto para aprender nuevas formas de trabajo como, en otras ocasiones, para confirmar lo que hago.

- Me ha beneficiado mucho ya que he podido ver otros enfoques diferentes $y$, en algún caso, me ha servido para reflexionar y cambiar mi percepción.

- La comunidad está muy bien ya que es una forma de ver, comparar, mejorar, cambiar tus "pensamientos" sobre algunas actividades y ayudar a los demás de la misma manera.

- Me ha dado más confianza y seguridad en mis evidencias.

- Ha sido un buen ejemplo para clarificar lo que se pedía, a través de las evidencias he aprendido mucho de otros compañeros y muchas de estas ideas las incorporaré en mi práctica educativa.

- La interacción nos ha enriquecido muchísimo, tanto en las evidencias como en las consultas que nos hemos ido haciendo.

- Por supuesto, trabajar dentro de la comunidad TeachersPro me ha beneficiado, ya que he tenido la posibilidad de conocer las buenas prácticas de otros compañeros y ha puesto a mi disposición recursos que me pueden ser de utilidad en el futuro.

- Realmente me ha sorprendido comprobar qué hacen mis compañeros del centro y 
cómo usamos instrumentos similares para evaluar o cómo afrontamos de distinta manera una misma situación.

- Quisiera haber tenido más interacción con compañeros a través de la plataforma, pero solo lo he tenido con una persona. Con ella, ha sido productivo.

- Gracias a la comunidad TeachersPro he podido ver gran variedad de evidencias, lo que me ha permitido sacar muchas ideas para implementar en mi práctica docente.

- Etc.

b) En relación con las 2020 habilidades realizadas por los docentes, podríamos resumir sus respuestas del siguiente modo:

1. En cuanto a los conocimientos sobre el tema que tenían los docentes, antes de comenzar esta habilidad, el $40 \%$ ya tenía algún conocimiento y el $60 \%$ tenía bastante o mucho, lo cual indica que las habilidades estudiadas fueron elegidas de acuerdo con el interés de los docentes por profundizar en su conocimiento.

2. En relación con lo que han cambiado algunas ideas que solían tener, en relación con el tema de esta habilidad, el $63 \%$ han cambiado mucho, a pesar de que el $60 \%$ ya tenía bastante idea sobre la habilidad.

3. Los problemas que me encontré mientras trabajaba en esta habilidad pude solucionarlos. El 86\% responde que lo hizo de manera muy positiva, lo cual muestra la versatilidad y utilidad de la herramienta.

4. Sobre lo que la práctica de esta habilidad ha beneficiado el aprendizaje de mis estudiantes, el $87 \%$ dice que lo ha hecho bastante o mucho, lo cual habla muy bien de la eficacia y la eficiencia de la herramienta.

5. En cuanto a lo que consideran que con esta experiencia ha mejorado su práctica docente, el $85 \%$ dice que ha mejorado mucho o bastante.

6. En cuanto al esfuerzo personal para abordar esta habilidad, el 78\% manifiesta que ha sido grande o muy grande, lo que significa que este aprendizaje les exige esfuerzo personal pero que influye, igualmente, en la mejora de su práctica $(85 \%)$, así como ha beneficiado a sus estudiantes $(87 \%)$.

7. Ha mejorado el domino de los conceptos relacionados con la habilidad desarrollada en el $87 \%$ de los casos ha mejorado bastante y mucho. Lo cual viene a corroborar y ser congruente con todas las opiniones anteriores.

8. En el desarrollo de la habilidad, me ha beneficiado la interacción con otros colegas de la comunidad TeachersPro. En un 66\% expresan que bastante o mucho, lo cual no deja de llamar la atención si lo comparamos con los resultados de las respuestas anteriores. Se podría pensar, tal como exponemos en otro apartado de este artículo, que aún necesitamos avanzar en este ámbito del compartir e interaccionar para superar el individualismo, pues, como es bien conocido, no existe mucha tradición en esta forma de aprender para enseñar.

9. En el desarrollo de la habilidad, me ha beneficiado la consulta y valoración de las evidencias aportadas por otros colegas de la Comunidad TeachersPro, en el $64 \%$ les ha beneficiado bastante o mucho.

\section{CONCLUSIONES Y PROSPECTIVA}

En consecuencia, y como hemos señalado antes, sería necesario saber que la planificación estratégica de la innovación pedagógica reflexiva a través de las comunidades profesionales de aprendizaje, tendrá verdadera influencia en la formación docente institucional, cuando seamos capaces de establecer sistemas de formación que permitan el desarrollo individual y colectivo del profesorado, que logren alinear las mochilas docentes (Malpica, 2013) de manera práctica, y su aplicación en el aula, para alcanzar las sinergias necesarias y desarrollar espacios comunes de aprendizaje docente que propicien la consecución de las metas que la institución educativa tiene establecidas.

Por tanto, en ese contexto de nuevos modelos formativos docentes, parece necesario encontrar un equilibrio entre el desarrollo de los entornos de aprendizaje personal y la formación docente institucional que bien planteados deben ser complementarios y no excluyentes; sobre todo, para que los planes estratégicos de innovación pedagógica reflexiva tengan un impacto positivo en la formación docente institucional deberían cumplir, entre otras, con las siguientes características:

1. Que la planificación estratégica de la innovación pedagógica reflexiva sea un instrumento de gestión escolar, que permita proyectar en el tiempo, una acción colegiada, para el logro de las metas institucionales. 
2. Que esta planeación estratégica será más eficaz y eficiente en la medida que posibilite $\mathrm{y}$ desarrolle la formación docente institucional.

3. Que la formación docente institucional debe apoyarse en modelos de desarrollo competencial que cumplan con las características señaladas; además, debe asegurar la alineación de hábitos docentes colectivos como forma de trabajo colegiado que aúna sinergias para el logro de las finalidades educativas institucionales.

4. Que la reflexión crítica sobre la práctica de los docentes, tanto individual como colectiva, sea el punto de partida para el logro de las comunidades profesionales de aprendizaje para una innovación pedagógica reflexiva y sostenible.

5. Que las plataformas de formación docente online como TeachersPro sean utilizadas como espacio formativo por su adecuación para mejorar los entornos personales de aprendizaje y el desarrollo docente en comunidades profesionales de aprendizaje.

En definitiva, como perspectivas de futuro, pensamos que la innovación pedagógica reflexiva y colaborativa adquiere todo su sentido, su eficacia e impacto, en la medida en la que se desarrolla dentro de comunidades profesionales de aprendizaje, que propician un desarrollo profesional docente que tiene como base la formación centrada en la escuela, para el desarrollo individual y colectivo de un sistema innovador, flexible y dinámico, capaz de proporcionar formación permanente en competencias profesionales docentes e institucionales, que den la respuesta adecuada para el logro de las finalidades educativas de cada institución.

\section{LIMITACIONES DEL ESTUDIO}

Esta reflexión pone de manifiesto la limitación aparente que supone analizar respuestas de docentes de ámbitos y contextos tan diferentes, lo cual podría convertirse en una gran riqueza si conseguimos un día poder contrastar las respuestas y opiniones de comunidades docentes procedentes de la misma institución, y que las instituciones educativas pertenezcan a realidades sociales, étnicas, culturales y políticas diferentes. Lo cual exigirá un estudio más amplio y sistemático de la innovación pedagógica reflexiva en comunidades profesionales de aprendizaje y su impacto en la formación docente institucional, que pueda seguir aportando información relevante para avanzar en esta línea de trabajo.

Otra limitación del análisis de estos resultados puede ser la voluntariedad de quienes responden, que ya se supone que tienen una motivación intrínseca por mejorar, cuando si queremos avanzar en el desarrollo de comunidades profesionales docentes debemos contar, conocer, analizar y valorar la opinión de la totalidad de los miembros de una institución. De otro modo, nos faltará tener presente, precisamente, la opinión de los que con frecuencia son parte del problema para avanzar en la buena dirección.

\section{REFERENCIAS}

Armijo, M. (2009). Manual de Planificación Estratégica e Indicadores de Desempeño en el Sector Público. Área de Políticas Presupuestarias y Gestión Pública ILPES/CEPAL. Recuperado de https://www.cepal.org/ilpes/noticias/paginas/3/38453/m anual_planificacion_estrategica.pdf

Domingo, À., y Gómez, $\overline{\mathrm{M}}$. V. (2014). La práctica reflexiva: bases, modelos e instrumentos. Madrid: Narcea.

Gairín Sallán, J. (2015). Las comunidades de práctica profesional: creación, desarrollo y evaluación. Wolters Kluwer Educación.

Imbernón, F. (2004). La profesión docente en el nuevo contexto educativo. En P. Carnicero Duque, P. Silva García, y T. Mentado Labao (Coords.). Nuevos Retos de la profesión docente (pp. 6-7). Barcelona: Universidad de Barcelona.

Malpica, F. (2013). Calidad de la práctica educativa. Barcelona: GRAO.

Monereo, C., y Pozo, J. I. (2001). ¿En qué siglo vive la escuela? Cuadernos de Pedagogía, 298, pp. 50-55.

Navareño, P. (2015). Descentralización con transparencia, liderazgo, calidad y pertinencia. Avances en Supervisión Educativa, (24), 1-37. DOI: https://doi.org/10.23824/ase.v0i24.9

Navareño, P. (2018). Práctica reflexiva en la formación profesional del docente. Ruta Maestra, Edición 22, 4952.

Navareño, P., y Rincón, J. (2017). Contribuciones de la Plataforma Online TeachersPro al desarrollo profesional del Entorno Personal de Aprendizaje. En J. RuizPalmero, J. Sánchez-Rodríguez y E. Sánchez-Rivas (Edit.). Innovación docente y uso de las TIC en educación. Málaga: UMA Editorial.

Robbins, S. P., y Coulter, M. (2005): Administración. México. Pearson Educación.

Senge, P., Cambron-McCabe, N., \& Lucas, T. (2002). Escuelas que aprenden. Bogotá: Norma.

Unesco (2015). Replantear la educación ¿Hacia un bien común mundial? París: Unesco. 